\title{
Construção e Validação da Escala de Imprevisibilidade Familiar na Infância (EIFI)
}

\author{
Construction and Validation of the Family Unpredictability Scale in Childhood
}

\author{
Anna Beatriz Carnielli Howat-Rodrigues, ${ }^{*}$, , Alexsandro Luiz De Andrade $^{b}$ \\ \& Rosana Suemi Tokumaru ${ }^{b}$ \\ ${ }^{a}$ Universidade de São Paulo, São Paulo, Brasil \& ${ }^{b}$ Universidade Federal do Espírito Santo, Vitória, Brasil
}

\begin{abstract}
Resumo
Este estudo teve o objetivo de construir e validar uma medida psicológica denominada de Escala de Imprevisibilidade Familiar na Infância (EIFI) para a população brasileira. Participaram 394 pessoas, sendo 158 adultos em conflito com a lei que já haviam passado pela prisão (média de idade $=34,23$ anos; $D P=10,17$ ), 122 estudantes universitários $(M=19,26 ; D P=2,06)$ e 114 mulheres com idade superior a 40 anos e escolaridade a partir de ensino médio $(M=51,19 ; D P=8,64)$. Foram conduzidos procedimentos de validade de conteúdo, face e construto. A análise fatorial gerou como produto um instrumento com estrutura de quatro dimensões (cuidado/apoio, recursos financeiros, alimentação e disciplina) com índices de confiabilidade satisfatórios para todas elas. Concluímos que a EIFI foi validada para a população brasileira apresentando-se no cenário nacional como um instrumento para medida de imprevisibilidade familiar na infância.

Palavras-chave: Imprevisibilidade familiar, infância, medida psicológica.

Abstract

This study aimed to construct and validate a psychological measure called Family Unpredictability Scale in Childhood for the Brazilian population. The study included 394 people: 158 adults in conflict with the law that had already been in prison $(M=34.23$ years; $S D=10.17), 122$ college students $(M=19.26 ; S D=2.06)$, and 114 women above 40 years old and, at least, high school schooling $(M=51.19 ; S D=8.64)$. Procedures of content, face and construct validity were conducted. The factor analysis generated an instrument structured in four dimensions (care/support, financial resources, food and discipline) with satisfactory reliability indexes for all of them. It was concluded that Family Unpredictability Scale in Childhood was validated to Brazilian population and that it can be used as an instrument to measure family unpredictability in childhood on the national scene.

Keywords: Family unpredictability, childhood, psychological measure.
\end{abstract}

A imprevisibilidade tem sido amplamente estudada a partir de medidas psicológicas (Alarcão \& Gaspar, 2007; Milliken, 1987; Ross \& Hill, 2000). Todavia, a variedade de definições para o termo pode dificultar a construção dos instrumentos utilizados para a mensuração do construto. A imprevisibilidade, também chamada de incerteza ambiental ou percepção de imprevisibilidade

\footnotetext{
"Este trabalho faz parte do projeto de pesquisa "Pessoas em conflito com a lei: História de vida e aspectos psicológicos", financiado pela Fundação de Amparo à Pesquisa do Espirito Santo (FAPES), Processo n. 4544 1855/09. Bolsa de mestrado Coordenação de Aperfeiçoamento de Pessoal de Nível Superior (CAPES) para A. B. C. Howat-Rodrigues. (Aluna do PPGP-UFES durante a realização do estudo).

Endereço para correspondência: Departamento de Psicologia Social e do Desenvolvimento, Universidade Federal do Espírito Santo, Av. Fernando Ferrari, s/n, Goiabeiras, Vitória, ES, Brasil 29060-900. E-mail: biacarnielli@yahoo.com.br, suemitokumaru@gmail.com e alexsandro.deandrade@yahoo.com.
}

ambiental, é um termo que pode receber várias interpretações e apresenta na literatura uma ampla gama de definições, adaptadas, frequentemente, do conceito de incerteza oferecido pelos campos da psicologia e da economia (Milliken, 1987). Dentre a confusão de definição e uso do construto, Milliken (1987), voltado para a teoria organizacional, reconhece que ora as definições são usadas para descrever o ambiente, ora para descrever as pessoas que percebem o ambiente.

O conceito de imprevisibilidade ambiental refere-se a características intrínsecas de um ambiente onde há falta de controle para regular e organizar os acontecimentos e disponibilidade sazonal de recursos, enquanto a percepção da imprevisibilidade ambiental é mais independente dos reais recursos disponíveis, pois preocupa-se com o nível de precisão com o qual se pode prever o futuro (Miller, 1996; Milliken, 1987; Ross \& Hill, 2002). A diferenciação entre os dois conceitos é difícil porque há influência mútua entre ambiente e percepção, já que a 
percepção, em primeira instância, depende das experimentações individuais em um determinado ambiente e, mais tarde, acaba influenciando na apreensão dos ambientes em geral (Hill, Ross, \& Low, 1997; Ross \& Hill, 2002). Faz-se importante pontuar que a imprevisibilidade não se refere à falta do recurso, mas sim, a incerteza sobre a disponibilidade do mesmo. Uma pessoa que trabalha com carteira assinada, por exemplo, ganhando um salário mínimo por mês, detém pouco recurso financeiro, mas é um recurso previsível, diferentemente de alguém que trabalha de forma autônoma ou informal e não pode precisar qual será sua renda em cada mês.

O construto imprevisibilidade também vem sendo tratado em termos desenvolvimentistas a partir da hipótese de que as percepções de imprevisibilidade obtidas no grupo familiar ou de criação funcionam como variáveis importantes para o desenvolvimento da criança (Ross \& Hill, 2000). Neste artigo, trabalhamos com a imprevisibilidade do ambiente familiar da criança considerando-a como uma variável importante para o desenvolvimento infantil (Ross \& Hill, 2000). Desta forma, tomaremos a imprevisibilidade a partir da percepção individual colocada em evidência por meio do relato dos indivíduos sobre a (im)previsibilidade familiar durante sua infância.

\section{O Conceito de Imprevisibilidade Familiar}

As organizações familiares encontram-se presentes em toda a história da humanidade e, acompanhando as mudanças socioeconômicas, vêm se modificando ao longo do tempo resultando em diferentes formas de vínculos afetivos (Milfont, Gouveia, \& Costa, 2006). A família pode ser entendida como fonte primária de suporte social a qual, dividindo uma esfera consaguínea e/ou afetiva comum, permite a aquisição de competências individuais na interação entre os seus membros (Pelzer \& Fernandes, 1997).

Ross e Hill (2000) chamam de imprevisibilidade familiar o nível de inconsistência do comportamento parental no cumprimento de suas responsabilidades (e.g. promoção de satisfação das necessidades básicas e sustentação dos sistemas de regras colocados para a criança). A percepção da imprevisibilidade ambiental durante a infância é tida como precursora de um modelo mental de imprevisibilidade, o qual auxilia o comportamento individual em diversos outros contextos, como, por exemplo, a tomada de decisão na vida adulta. Este modelo permeia a percepção individual sobre a disponibilidade de recursos (e.g. de subsistência, financeiros, de cuidados, entre outros) com a qual se pode contar no ambiente (Ross \& Hill, 2002).

O modelo de imprevisibilidade de Ross e Hill (2002) indica a percepção de previsibilidade dos recursos como sendo mais importante do que a presença dos próprios recursos. Além disso, leva-se em conta que fatores como a expectativa de vida individual, a percepção de perigo ambiental (Hill, Jenkins, \& Farmer, 2008; Hill et al., 1997), a ocorrência de separação dos pais e o baixo nível socioeconômico (Hill et al., 1997) auxiliem para uma percepção de imprevisibilidade ambiental. Ocorrendo no contexto infantil, estas variáveis contribuem para uma maior inconsistência no comportamento parental e, portanto, uma maior percepção de imprevisibilidade da criança sobre seu ambiente.

\section{Medidas Psicológicas de Imprevisibilidade Familiar}

Em relação ao desenvolvimento de instrumentos de imprevisibilidade familiar destacam-se no contexto internacional os trabalhos de Alarcão e Gaspar (2007), Ross e Hill (1995, 2000) e Ross e McDuff (2008), os quais focam a percepção individual de imprevisibilidade do ambiente familiar. A fim de acessar esquemas de imprevisibilidade familiar, Ross e Hill (1995) perguntaram a jovens adultos, a partir da mensuração em escalas do tipo Likert, as sentenças seguintes: (a) Eu tenho uma boa idéia (previsão) sobre o que irá acontecer em minha vida, (b) Basicamente, o mundo é um lugar previsível, e (c) Eu sei o que esperar das pessoas que fazem parte da minha vida. Estas três sentenças ganharam o nome inicial de escala de crenças de imprevisibilidade, obtendo um coeficiente de confiabilidade (alfa de Cronbach) de 0,80 e correlacionando-se positivamente com o lócus externo de controle de causa de imprevisibilidade.

Interessadas na percepção de pais sobre a imprevisibilidade do ambiente dos filhos, Ross e Hill (2000) desenvolveram instrumento escalar nomeado de Family Unpredictability Scale (FUS), validado com população norte-americana. As autoras pediram a pais com filhos de 2 a 18 anos, adotivos ou biológicos, que respondessem, em escala Likert de 5 pontos, sobre o quanto os 198 itens (formulados por 7 pesquisadores especialistas dentro de categorias como recreação, hobbies, dinheiro, rotinas, disciplina, afeição, necessidades físicas, férias, finais de semana, trabalho, educação e aperfeiçoamento, alimentação, etc.) relacionados a previsibilidade ou imprevisibilidade, aplicavam-se à sua família, mais especificamente às crianças da família. A FUS resultou em um total de 23 itens distribuídos em quatro fatores: sete itens no fator disciplina, sete no fator cuidado/apoio, cinco no fator alimentação e três no fator recursos financeiros. A dimensão disciplina correspondeu a comportamentos de inconsistência parental no estabelecimento e manutenção de regras; cuidado/apoio refletiu os comportamentos relacionados à inconsistência parental no fornecimento de cuidados à criança diante de suas necessidades de apoio emocional (conforto, proteção, afeto) e de apoio no enfrentamento de situações estressantes; alimentação correspondeu a comportamentos relacionados à incerteza da hora das refeições e quem as compartilhava e, finalmen- 
te, recursos financeiros refletiu a incerteza em relação à previsão de recursos financeiros para o pagamento de gastos e obtenção de bens (Ross \& Hill, 2000, 2002).

Em estudo de validação, a partir da tradução da FUS para a população portuguesa, Alarcão e Gaspar (2007), encontraram fragilidades do instrumento com altos índices de saturação para os itens de cada componente, desta forma, os dados apresentaram distribuição fatorial diversa da proposta original de Ross e Hill (2000). As autoras apontam como possíveis vieses da pesquisa a não aleatoriedade da amostra, a possibilidade da influência da desejabilidade social nas respostas dadas pelos participantes e as diferenças culturais entre a população norteamericana e portuguesa não contemplada pela simples tradução da escala. A simples tradução da escala pode ter sido um fator importante para a diferença entre os fatores encontrados por Alarcão e Gaspar (2007) e Ross e Hill (2000). De acordo com Erthal (2001), os elementos de validade, fidedignidade, normatização e padronização devem ser garantidos em diferentes contextos culturais a fim de possibilitar generalizações.

Ross e McDuff (2008) adaptaram a FUS para a investigação da percepção de imprevisibilidade do contexto familiar durante a infância. A Retrospective Family Unpredictability Scale (R-FUS) consiste de 28 itens, distribuídos nos mesmos 4 fatores já descritos para a FUS (disciplina, alimentação, cuidado/apoio e recursos financeiros) e também avaliados em escala Likert de 5 pontos. As autoras validaram a escala para a população norteamericana com a obtenção de resultados satisfatórios.

Em contexto brasileiro não encontramos produção sobre o tema da imprevisibilidade familiar. Porém, temas como família, comportamento parental, desenvolvimento infantil e garantia de recursos alimentares são assuntos amplamente discutidos na Psicologia e nas Ciências Humanas em geral, existindo nestes campos vários estudos com medidas que, frequentemente, trazem em sua estruturação informações sobre características referentes à imprevisibilidade familiar. Destacam-se a Escala Brasileira de Insegurança Alimentar (EBIA; Segall-Corrêa et al., 2003), o Inventário de Recursos do Ambiente Familiar (Marturano, 2006), o Questionário Sobre Traumas na Infância (Grassi-Oliveira, Stein, \& Pezzi, 2006) e o Questionário de Estilos Parentais (Boeckel \& Castelá Sarriera, 2005). A EBIA avalia a garantia familiar de recursos alimentares e é uma medida adaptada dos estudos de Radimer, Olson, Greene, Campbell e Habicht (1992) e validada para a população brasileira por Segall-Corrêa et al. (2003). Ela consiste em 15 afirmativas, com resposta do tipo sim ou não e sobre a frequência com que o evento mensurado ocorreu nos últimos três meses, cada resposta afirmativa corresponde a 1 ponto. A pontuação 0 corresponde à segurança alimentar, variações entre 1-5 pontos em famílias com presença de menores de idade ou 1-3 em famílias sem menores de idade são interpretadas como insegurança leve, 6-10 ou 4-6 como insegurança moderada e 11-15 ou 7-8 como insegurança grave.
Para caracterização do contexto familiar encontramos o Inventário de Recursos do Ambiente Familiar (Marturano, 2006) e o Questionário Sobre Traumas na Infância (Grassi-Oliveira et al., 2006). Aquele é um instrumento criado para a população brasileira por Marturano (2006) com o objetivo de levantamento de recursos no contexto familiar influenciadores do aprendizado acadêmico da criança nos anos de ensino fundamental. Baseado no modelo bioecológico do desenvolvimento, este instrumento compõem-se de 10 questões direcionadas as mães ou cuidadores de crianças, aplicadas sob forma de entrevista semi-estruturada, portanto, não geram escore. Os itens referem-se aos recursos que sinalizam estabilidade familiar e às práticas parentais que promovem proximidade entre criança e escola. Já o Questionário Sobre Traumas na Infância foi adaptado do Childhood Trauma Questionnaire (CTQ; Bernstein \& Fink, 1998; Bernstein et al., 2003) por Grassi-Oliveira et al. (2006), pode ser encontrado em versão completa com 28 itens e em versão sintetizada com 25 itens. Os itens são relacionados a experiências da infância e adolescência, distribuem-se em 5 dimensões clínicas (abuso físico, sexual e emocional e negligencia física e emocional) e são avaliados em escala Likert de 5 pontos. Em relação ao estilo parental, o Questionário de Estilos Parentais ou Parental Authoritative Questionnaire (PAQ; Boeckel \& Castellá Sarriera, 2005; Buri, 1991) é um instrumento composto de 30 afirmativas sobre a percepção dos filhos da educação recebida durante a infância. $\mathrm{O}$ questionário é formado por 3 subescalas, com 10 itens cada uma, referentes aos estilos parentais autoritário, autorizante e permissivo, avaliados em uma escala Likert de 5 pontos. É importante salientar que em estudo brasileiro os resultados apontaram nível de consistência interna satisfatório nos 3 fatores mensurados, detectando possibilidade de aplicação do instrumento em tal contexto (Boeckel \& Castellá Sarriera, 2005).

De forma geral e, principalmente no que se refere ao cenário brasileiro, constatamos pouca padronização em medidas de imprevisibilidade familiar, dificultando o estudo de tal construto que ainda é fonte de confusões teóricas e de ausência de definições consensuais. Levando-se em conta a diversidade cultural e a construção de instrumentos voltados, principalmente, para populações com alta escolaridade, faz-se importante a construção, validação e padronização da medida a fim de garantir a confiabilidade da mesma. O objetivo deste estudo foi à estruturação (construção e validação) de uma medida psicológica denominada de Escala de Imprevisibilidade Familiar na Infância (EIFI) para a população brasileira. Salientamos que esta pesquisa é parte integrante de um projeto maior o qual relaciona variáveis de imprevisibilidade familiar durante a infância, propensão ao risco e desconto do futuro (tendência ao adiantamento de ganhos menores no presente a espera de ganhos maiores no futuro). 
Howat-Rodrigues, A. B. C., De Andrade, A. L. \& Tokumaru, R. S. (2012). Construção e Validação da Escala de Imprevisibilidade Familiar na Infância (EIFI).

\section{Método}

Este estudo teve um caráter quantitativo e constou de duas etapas para o desenvolvimento da medida: a primeira dedicada à construção do instrumento e a segunda relacionada à análise das propriedades psicométricas da medida, incluindo a análise empírica e estatística da EIFI, bem como verificação de propriedades discriminantes da medida.

\section{Primeira Etapa: Construção da Escala}

Tradução e adaptação dos itens. O instrumento desenvolvido baseou-se no estudo de Ross e McDuff (2008), anteriormente apresentado. Com a finalidade de evidenciar empiricamente o construto, identificar a dimensionalidade do mesmo e verificar a existência de itens prováveis em fontes nacionais e internacionais realizamos levantamento bibliográfico nas bases de pesquisa Lilacs, Index Psi, Scielo, Google Acadêmico e Periódicos Capes sobre o tema imprevisibilidade familiar e sua multidimensionalidade (cuidado/apoio, recursos financeiros, disciplina e alimentação).

A Retrospective Family Unpredictability Scale (R-FUS; Ross \& McDuff, 2008), foi traduzida por um especialista no idioma inglês, optamos já pela adaptação semântica dos itens traduzidos ao contexto brasileiro. Os itens foram reformulados, acrescidos e adaptados ao construto imprevisibilidade familiar com o auxílio de instrumentos brasileiros: Escala Brasileira de Insegurança Alimentar (Segall-Corrêa et al., 2003), o Inventário de Recursos do Ambiente Familiar (Marturano, 2006), o Questionário Sobre Traumas na Infância (Grassi-Oliveira et al., 2006) e o Questionário de Estilos Parentais (Boeckel \& Castellá Sarriera, 2005). Para a adequação dos itens à população brasileira contamos ainda com o auxílio de um especialista na área de psicometria.

Validade de face e de conteúdo. Dois critérios qualitativos foram adotados para avaliar a qualidade geral do instrumento a partir de seus itens: a validade de face e a validade de conteúdo. O procedimento de validade de face objetivou verificar a compreensão de cada item para todos os membros da população a qual se destinava, a partir de consulta aos participantes destes grupos (Pasquali, 1998). Já a análise de conteúdo permitiu testar a pertinência do conteúdo dos itens as dimensões da escala que eles se propõem a mensurar (Pasquali, 1998).

Para atingir o aspecto de validade de face o instrumento foi submetido à avaliação de três representantes de menor escolaridade da nossa amostra, os quais consideramos apresentarem potencialmente maior dificuldade de compreensão semântica. Os itens da escala foram lidos de forma individual junto com os participantes, após ciência do objetivo da tarefa. Os mesmos foram instruídos a responder a escala, explicando o que haviam entendido do item e apontando qualquer dificuldade de compreensão da sentença ou de palavra dentro da sentença. Além disso, o pesquisador esteve atento ao enten- dimento geral dos itens e das palavras diante das quais os participantes apresentavam maior hesitação na resposta e da escala Likert proposta.

Para alcance da validade de conteúdo dos itens, quatro especialistas foram convidados para avaliação do instrumento. Todos possuíam título de doutor ou obtenção de título em andamento e apresentavam afinidade com a utilização de escalas e/ou com o tema de pesquisa. A tarefa dos juízes consistiu em julgar o pertencimento de cada um dos 43 itens às dimensões de disciplina, alimentação, cuidado/apoio e recursos financeiros da escala de imprevisibilidade familiar. Para isso, os mesmos receberam através de seus endereços eletrônicos pessoais um convite para a contribuição no desenvolvimento da escala na etapa de validade de conteúdo.

Estudo piloto. A partir das considerações de mudanças no processo de validade de face e de conteúdo, o instrumento reformulado foi submetido a um procedimento de estudo piloto, cujo objetivo foi analisar a pertinência e entendimento dos itens propostos para as amostras estudadas. Nove pessoas foram convidadas a participar do estudo piloto. Quatro dos participantes possuíam escolaridade mínina correspondente ao ensino médio e foram convidados a ler e responder os itens sem interferência da pesquisadora, ou seja, de forma autoaplicável. Com os outros cinco participantes a aplicação ocorreu em forma de entrevista realizada pela pesquisadora devido à baixa escolaridade (até ensino fundamental), o que dificultava a compreensão do que liam. Foi solicitado que, caso não compreendessem alguma questão, anotassem ou falassem suas observações, dando sugestões.

\section{Segunda Etapa: Análise das Propriedades \\ Psicométricas e Discriminantes da EIFI}

Participantes. Seguindo critérios sobre o número de participantes necessários para realização de procedimentos estatísticos de validação de uma escala, consideramos uma representatividade de 5 participantes por item da escala (Laros, 2005; Pasquali, 1999).

Participaram, nesta etapa, um total de 394 pessoas, distribuídos em três grupos amostrais, destes, (a) 149 homens e nove mulheres eram adultos em conflito com a lei, (b) 114 eram mulheres com idade igual ou superior a 40 anos e escolaridade a partir de ensino médio e (c) 108 homens e 14 mulheres eram estudantes universitários. Os grupos amostrais foram selecionados de acordo com critérios de faixa etária e sexo, reportados na literatura como influentes na propensão ao risco e desconto do futuro individual - homens jovens seriam a população com maiores propensões ao risco e desconto do futuro (Daly \& Wilson, 2001; Hill et al., 1997; Steinberg, 2004). A distribuição heterogênea de sexos no grupo de adultos em conflito com a lei relaciona-se a diferença de distribuição entre homens e mulheres nesta população, de maioria masculina.

Todos os jovens tinham escolaridade de ensino superior incompleto, não tinham respondido processo criminal, 
apresentaram média de idade de 19,26 anos $(D P=2,06)$. Assim como os jovens universitários, as mulheres acima de 40 anos não tinham respondido processo criminal. Declararam escolaridade de ensino superior ou médio ( $57 \%$ e $43 \%$, respectivamente) e média de idade de 51,19 anos $(D P=8,64)$. Os adultos em conflito com a lei apresentaram em sua maioria escolaridade de ensino fundamental (65,8\% Fundamental, 27,8\% Médio, 3,8\% Superior, 2,5\% Analfabeto) e idade média de 34,23 $\operatorname{anos}(D P=10,17)$.

Instrumentos. A EIFI consistiu inicialmente de 40 itens, autoavaliados em escala Likert de 5 pontos $(1=$ discordo totalmente; $5=$ concordo totalmente). Assim como a R-FUS, a EIFI foi construída baseada na multidimensionalidade de quatro fatores: alimentação, recursos financeiros, cuidado/apoio e disciplina. A medida teve como instrumentos de subsídio (a) a R-FUS (Ross $\&$ Hill, 2002) traduzida e adaptada, (b) a EBIA (SegallCorrêa et al., 2003), (c) o Inventário de Recursos do Ambiente Familiar (Marturano, 2006), (d) o Questionário Sobre Traumas na Infância (Grassi-Oliveira et al., 2006) e (e) o Questionário de Estilos Parentais (Boeckel \& Castellá Sarriera, 2005). Além da EIFI, aplicamos questionário sociodemográfico com itens de caracterização dos participantes (sexo, idade, escolaridade, escores do Critério de Classificação Econômica Brasil - CCEB). O CCEB é utilizado originalmente de forma categórica (classes econômica $\mathrm{A}$ à $\mathrm{D}$ ), mas aqui foi utilizado como medida contínua sendo atribuída uma pontuação $(0$ a 46 pontos) para cada participante sobre seu poder de compra.

Aspectos éticos e procedimento de coleta de dados. Esta pesquisa cumpriu com os procedimentos éticos em pesquisa e foi autorizada pelo Comitê de Ética em Pesquisa da Universidade Federal do Espírito Santo sob o número 089/09. Os participantes foram informados sobre a pesquisa pelo pesquisador responsável e consentiram na participação através de Termo de Consentimento Livre e Esclarecido.

Adultos em conflito com a lei foram convidados a participar da pesquisa por meio de uma Vara de Penas e Medidas Alternativas, mediante autorização do Juiz de Direito responsável. Os jovens universitários foram convidados em sala de aula. A entrada em salas de aula foi permitida pelo responsável pela instituição de ensino superior no qual a coleta foi realizada. As mulheres acima de 40 anos foram convidadas mediante contatos pessoais da pesquisadora.

A EIFI tem caráter autoaplicável, todavia, este caráter obedeceu critérios de escolaridade: a autoaplicação ocorreu quando o participante tinha escolaridade a partir de ensino médio incompleto e a aplicação pela pesquisadora ocorreu em casos de menor escolaridade. Mulheres com mais de 40 anos e adultos em conflito com a lei participaram de sessões individuais de aplicação enquanto os jovens universitários participaram de sessões coletivas.

Análise de dados. Os dados foram tratados com auxílio do pacote estatístico SPSS (Statistical Package for the
Social Sciences), versão 13. Além de estatística descritiva (frequência, porcentagem, média) para construção da escala e caracterização dos participantes, utilizamos análises fatoriais exploratórias com rotação varimax e cálculo de confiabilidade alfa de Cronbach para obter as estruturas fatoriais da escala psicológica resultante. Utilizamos ainda análise comparativa entre grupos a partir de análise de variância multivariada (MANOVA) para verificação de aspectos de validade discriminante da medida.

\section{Resultados}

\section{Primeira Etapa: Construção da Escala}

O instrumento proposto na etapa de validação de face, após tradução, adaptação e acréscimos baseados na literatura, foi composto por 49 itens, 12 correspondendo à categoria disciplina, 11 à categoria alimentação, 15 à cuidado/apoio e 11 à recursos financeiros. Após participação de três representantes da amostra de adultos em conflito com a lei, seis itens foram excluídos devido à difícil compreensão e adequação dos mesmos (e.g. "Eu sabia o que as pessoas da minha família esperavam de mim diante de uma situação"). Doze itens sofreram adequação semântica, sentenças com as expressões "era difícil de prever", "era imprevisível" e "era previsível" foram substituídas pela expressão "Eu tinha certeza", optando-se pela forma positiva da questão (e.g. "Era difícil prever se teríamos recursos financeiros." transformou-se em "Minha família tinha certeza de que teria recursos financeiros").

A validação de conteúdo foi realizada com instrumento composto por 43 itens. Na avaliação dos juízes, $68 \%$ dos itens obtiveram índice de concordância de 100\%, perfazendo um total de 29 itens. Sete itens (17\%) apresentaram índice de concordância entre $80 \%$ e $99 \%$. Quatro itens apresentaram concordância entre $60 \%$ e $79 \%$ e, nestes casos, optamos pela manutenção dos que apresentaram tais índices quando este julgamento correspondia à dimensão esperada pelos pesquisadores. Três itens foram excluídos por não atingirem um índice de concordância satisfatório de pelo menos 60\% ("Era difícil prever como as pessoas da minha família iriam reagir diante das situações"; "Houve momentos em que não sabíamos se teríamos o que comer nas próximas refeições"; "Alguém da minha família ou toda a família tinha que fazer apenas uma refeição por dia porque tínhamos pouco alimento em casa").

Em resumo, foram excluídos três itens, correspondendo a $6 \%$ do total de 43 itens iniciais. Dois itens foram redimensionados passando da dimensão alimentação para a dimensão recursos financeiros. Os itens mantidos distribuíram-se entre as dimensões da seguinte maneira: dez itens em disciplina, seis itens em alimentação, 11 itens em cuidado/apoio e 13 itens em recursos financeiros, perfazendo um total de 40 itens. Após estudo piloto não foi necessário reestruturar a escala ou eliminar itens já que todos foram claramente compreendidos. 
Howat-Rodrigues, A. B. C., De Andrade, A. L. \& Tokumaru, R. S. (2012). Construção e Validação da Escala de Imprevisibilidade Familiar na Infância (EIFI).

Segunda Etapa: Análise das Propriedades

Psicométricas e Discriminantes da EIFI

A análise dos componentes principais da EIFI foi realizada inicialmente para verificar a adequação da matriz de dados ao procedimento de análise fatorial e a quantidade de fatores a serem extraídos. A mesma apresentou valores considerados aptos para realização dos procedimentos fatoriais, o KMO obteve valor de 0,85 e o Teste de esfericidade de Bartlett foi significativo 5713,291 ( $g l=780, p<0,001)$, valores que satisfazem condições para o procedimento fatorial (Pasquali, 2005).

Para decisão do número de fatores a serem extraídos optamos pelo critério do gráfico de sedimentação de Catell, bem como elementos ligados a interpretação teórica do construto. $\mathrm{O}$ gráfico de sedimentação sugeriu a solução de quatro fatores, assim como a teoria, explicando $35,29 \%$ da variância total dos dados.

Definido o número de fatores, procedemos à realização da extração dos fatores pelo método fatoração dos eixos principais (PAF), com rotação do tipo varimax, justificada pela suposição de relação de independência entre os fatores, e cálculos de confiabilidade. Na Tabela 1 estão apresentados os 27 itens, suas respectivas comunalidades, quantidade de itens, a variância explicada por fator e os índices de confiabilidade do tipo alfa de Cronbach de cada um deles, na fatoração do eixo principal. Cada fator foi designado de acordo com a característica geral dos itens compreendidos resultando, desta forma, nas seguintes dimensões: Cuidado/apoio (Fator 1), Recursos Financeiros (Fator 2), Alimentação (Fator 3) e Disciplina (Fator 4).

Tabela 1

Fatores Extraidos, Cargas Fatoriais, Comunalidades, Quantidade de Itens, Variância Explicada por Cada Fator, Índices de Confiabilidade do Tipo Alfa de Cronbach da EIFI

\begin{tabular}{|c|c|c|c|c|c|}
\hline & \multicolumn{5}{|c|}{ Fator } \\
\hline & 1 & 2 & 3 & 4 & $h^{2}$ \\
\hline Eu tinha certeza de que minha família cuidaria de mim. & 0,79 & & & & 0,65 \\
\hline Eu tinha certeza de que minha família me daria apoio se eu precisasse. & 0,76 & & & & 0,59 \\
\hline Eu sabia que minha família estaria presente para cuidar de mim. & 0,71 & & & & 0,55 \\
\hline Eu me sentia amado pela minha família. & 0,64 & & & & 0,43 \\
\hline Eu sabia que minha família estaria presente para me proteger. & 0,62 & & & & 0,43 \\
\hline $\begin{array}{l}\text { Quando eu me machucava, eu sabia que poderia recorrer às pessoas } \\
\text { da minha família para ter os primeiros cuidados. }\end{array}$ & 0,61 & & & & 0,38 \\
\hline Eu sabia que eu era importante para minha família. & 0,61 & & & & 0,39 \\
\hline $\begin{array}{l}\text { Eu sabia que as pessoas da minha família cuidavam umas das outras. } \\
\text { Quando eu estava chateado eu sabia que poderia procurar consolo }\end{array}$ & 0,54 & & & & 0,35 \\
\hline com a minha família. & 0,54 & & & & 0,34 \\
\hline Eu sabia que minha família se preocupava com minha educação. & 0,54 & & & & 0,35 \\
\hline $\begin{array}{l}\text { Se uma nova regra fosse decidida para manter a disciplina da casa, } \\
\text { eu sabia que minha família iria me explicar de forma clara. }\end{array}$ & 0,49 & & & & 0,27 \\
\hline $\begin{array}{l}\text { Houve momentos na minha casa que faltava dinheiro para comprar } \\
\text { coisas de necessidade básica (higiene, vestuário, etc.) }\end{array}$ & & 0,83 & & & 0,70 \\
\hline $\begin{array}{l}\text { Houve momentos na minha infância em que tínhamos pouco dinheiro } \\
\text { para viver. }\end{array}$ & & 0,75 & & & 0,57 \\
\hline $\begin{array}{l}\text { Na minha casa, não sabíamos se haveria comida para as refeições diárias. } \\
\text { Na minha infância, minha família não sabia ao certo como pagaria }\end{array}$ & & 0,66 & & & 0,46 \\
\hline as contas de cada mês. & & 0,63 & & & 0,43 \\
\hline $\begin{array}{l}\text { Eu e/ou outras crianças da minha casa tivemos que começar } \\
\text { a trabalhar cedo. }\end{array}$ & & 0,62 & & & 0,43 \\
\hline $\begin{array}{l}\text { Minha família tinha a preocupação de que os alimentos acabassem } \\
\text { antes de poder comprar mais. }\end{array}$ & & 0,59 & & & 0,37 \\
\hline $\begin{array}{l}\text { Na minha infância, meus familiares costumavam ter empregos } \\
\text { informais (sem carteira assinada). }\end{array}$ & & 0,54 & & & 0,31 \\
\hline $\begin{array}{l}\text { Na infância, houve pessoas da minha família que ficaram desempregadas. } \\
\text { Na minha casa o horário que o jantar era servido normalmente }\end{array}$ & & 0,50 & & & 0,31 \\
\hline era o mesmo todos os dias. & & &, 72 & & 0,54 \\
\hline $\begin{array}{l}\text { Na minha casa o horário em que o almoço era servido normalmente } \\
\text { era o mesmo todos os dias. }\end{array}$ & & &, 69 & & 0,50 \\
\hline $\begin{array}{l}\text { Na minha infância, de segunda a sexta-feira, eu sabia que } \\
\text { as mesmas pessoas iriam se sentar à mesa para jantar. }\end{array}$ & & &, 64 & & 0,51 \\
\hline
\end{tabular}


Na minha casa o horário das refeições era diferente a cada dia.

$\mathrm{Na}$ minha casa, eu sabia quem estaria presente na hora das refeições.

$\mathrm{Na}$ minha casa, de segunda a sexta-feira, as mesmas pessoas costumavam estar reunidas no café da manhã.

Na minha casa a disciplina que os meus familiares me davam

era inconsistente (ora me disciplinavam, ora não).

$\mathrm{Na}$ minha casa, as regras que os meus familiares me davam

eram inconsistentes (ora me davam regras, ora não).

Número de itens

\% da variância explicada

alfa de Cronbach

\begin{tabular}{|c|c|c|c|c|}
\hline & & $-0,57$ & & 0,45 \\
\hline & & 0,56 & & 0,34 \\
\hline & & 0,42 & & 0,24 \\
\hline & & & 0,72 & 0,60 \\
\hline & & & 0,67 & 0,51 \\
\hline 11 & 8 & 6 & 2 & \\
\hline 13,11 & 11,03 & 6,95 & 4,92 & \\
\hline 0,88 & 0,85 & 0,80 & 0,78 & \\
\hline
\end{tabular}

Foi tomado o critério apontado para consideração de relevância de cargas fatoriais os itens com carga igual ou superior a 0,40 (Dancey \& Reidy, 2006). Dos 40 itens, treze foram excluídos por apresentarem cargas fatoriais menores que 0,40 e, consequentemente, representarem de forma menos relevante os fatores extraídos.

Os outros 27 itens, distribuídos em 4 fatores, corresponderam à descrição proposta por Ross e Hill (2000, 2002) em versão norte-americana da EIFI. Desta forma, o Fator 1, nomeado de cuidado/apoio, refletiu os comportamentos relacionados à inconsistência parental no fornecimento de cuidados à criança diante de suas necessidades de apoio emocional (conforto, proteção, afeto) e de apoio no enfrentamento de situações estressantes. O Fator 2, nomeado de recursos financeiros, refletiu a incerteza em relação à previsão de recursos financeiros para o pagamento de gastos e obtenção de bens. O Fator 3, chamado de alimentação, correspondeu a comportamentos relacionados à incerteza da hora das refeições e quem as compartilhava e, finalmente, o Fator 4, denominado disciplina correspondeu a comportamentos de inconsistência parental no estabelecimento e manutenção de regras. $\mathrm{O}$ valor do alfa de Cronbach foi considerado satisfatório para os 4 fatores por terem sido maiores que 0,70 , indicando confiabilidade das subescalas resultantes (Pasquali, 2005).

A fim de investigar fatores discriminantes na percepção de imprevisibilidade os grupos foram diferenciados de acordo com a literatura em relação à expectativa de vida - alto ou baixo índice de mortalidade e de perigo ambiental (Hill et al., 2008; Hill et al., 1997) e o nível socioeconômico (Hill et al., 1997), representado aqui pela pontuação no CCEB. Houve diferença significativa de expectativa de vida, $F(2,369)=9,22, p<0,0001$, e pontuação no $\mathrm{CCEB}, F(2,380)=93,00, p<0,0001$. O post - hoc indicou que adultos em conflito com a lei diferenciaramse estatisticamente dos outros dois grupos na expectativa de vida e no CCEB apresentando as menores médias: $73,73(D P=18,83)$ e $17,9(D P=6,25)$, respectivamente. Enquanto mulheres e jovens apresentaram média de expectativa de vida de $81,81(D P=17,30)$ e $82,47(D P$ $=19,34)$, respectivamente e média no CCEB de $28,72(D P$ $=6,79)$ e $27,56(D P=8,44)$.

Em conformidade com as diferenças acima apontadas, os grupos também diferiram quanto à imprevisibilidade familiar durante a infância no teste MANOVA (Tabela 2).

Tabela 2

Análises de Post Hoc (método Bonferroni) de Comparações entre Grupos para as Variáveis de Imprevisibilidade Familiar Durante a Infância (MANOVA)

\begin{tabular}{|c|c|c|c|c|c|c|}
\hline \multicolumn{7}{|c|}{ Dimensões de Imprevisibilidade Familiar na Infância (EIFI) } \\
\hline & & & Cuidado/Apoio & Recursos financeiros & Alimentação & Disciplina \\
\hline \multirow{3}{*}{ 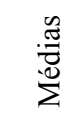 } & & Mulher & 1,537 & 2,783 & 1,759 & 1,474 \\
\hline & & Adulto & 1,518 & 3,520 & 2,345 & 2,364 \\
\hline & & Jovem & 1,423 & 1,879 & 2,337 & 1,627 \\
\hline \multirow{5}{*}{\multicolumn{2}{|c|}{ 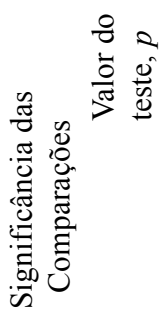 }} & & \multicolumn{4}{|c|}{$F(8,776)=30,776, p<0,0001 ;$ lambda de Wilks $=0,58$} \\
\hline & & & $\begin{array}{l}F(2,391)=1,10 \\
\quad p=0,34\end{array}$ & $\begin{array}{c}F(2,391)=23,86 \\
\quad p<0,0001\end{array}$ & $\begin{array}{l}F(2,391)=13,88 \\
p<0,0001\end{array}$ & $\begin{array}{c}F(2,391)=22,60, \\
p<0,0001\end{array}$ \\
\hline & & Mulher - Adulto & 1,000 & $<0,0001^{*}$ & $<0,0001 *$ & $<0,0001 *$ \\
\hline & & Mulher - Jovem & 0,534 & $<0,0001^{*}$ & $<0,0001^{*}$ & 0,965 \\
\hline & & Adulto - Jovem & 0,666 & $<0,0001^{*}$ & 1,000 & $<0,0001^{*}$ \\
\hline
\end{tabular}

\footnotetext{
* Significância $<0,0001$.
} 
Howat-Rodrigues, A. B. C., De Andrade, A. L. \& Tokumaru, R. S. (2012). Construção e Validação da Escala de Imprevisibilidade Familiar na Infância (EIFI).

As diferenças ocorreram em relação à impre-visibilidade de recursos financeiros, alimentação e disciplina. A Tabela 2 apresenta as análises subsequentes (post hoc) à MANOVA do tipo de comparação entre os grupos (pairwise comparisons, ajustadas pelo método de Bonferroni) para as quatro variáveis de imprevisibilidade.

Todos os grupos se diferenciaram estatisticamente na imprevisibilidade de recursos financeiros, tendo os adultos em conflito com a lei apresentado às maiores médias e os jovens as menores médias. Em imprevisibilidade de alimentação, o grupo de mulheres se diferenciou dos outros dois grupos apresentando as menores médias. Finalmente, o grupo de adulto em conflito com a lei se diferenciou dos outros dois apresentando as maiores médias de imprevisibilidade de disciplina.

\section{Discussão e Considerações Finais}

Confirmando estudos internacionais (Ross \& Hill, 2000, 2002), os resultados do presente estudo apontam para uma escala de imprevisibilidade familiar composta de quatro dimensões: cuidado/apoio, recursos financeiros, alimentação e disciplina. Estas dimensões foram constituídas por onze, oito, seis e dois itens, respectivamente, com cargas fatoriais satisfatórias. Não se pode afirmar, no entanto, que as quatro dimensões aqui identificadas constituemse as mais importantes ou mesmo as únicas no âmbito da imprevisibilidade. Além destes fatores aqui expressos, outras dimensões de imprevisibilidade familiar podem ser pensadas tais como recreação, hobbies, necessidades físicas, entre outras (Ross \& Hill, 2000). Consideramos, contudo, que os objetivos deste estudo foram contemplados a partir do desenvolvimento de uma escala que mensura de forma satisfatória componentes de imprevisibilidade familiar.

Diferentemente do trabalho de Alarcão e Gaspar (2007) com população portuguesa, sugerimos que a EIFI sustentou o modelo de quatro domínios de imprevisibilidade devido às etapas de validação de face e de conteúdo as quais permitiram maior controle sobre componentes culturais que influenciam o desenvolvimento de modelos de imprevisibilidade (Pasquali, 2001, 2007; Urbina, 2007). Há de se considerar, contudo, a influência do fator cultural. Neste sentido, à distribuição dos itens esperados na dimensão disciplina agruparam-se com a dimensão cuidado/apoio e dos itens esperados na dimensão alimentação agruparam-se com itens da dimensão recursos financeiros. Nos quatro casos os conteúdos dos itens foram considerados aceitáveis para fazerem parte da dimensão com a qual se agruparam.

Quanto aos itens excluídos (13 itens) por apresentarem carga fatorial insuficiente, seis deles eram esperados na dimensão disciplina, cinco na dimensão de recursos financeiros e dois na dimensão cuidado e apoio. Entendemos que o elemento cultural tenha contribuído para a baixa frequência de itens na dimensão final de disciplina e a migração de alguns itens de disciplina para a dimensão cuidado/apoio. Em um total de dez itens esperados no fator disciplina, apenas dois apresentaram cargas fatoriais satisfatórias nesta dimensão e outros dois carregaram em cuidado/apoio. A dimensão disciplina corresponde à inconsistência parental no estabelecimento e manutenção de regras para com suas crianças (Ross \& Hill, 2000). Todavia, ampliando este conceito tem-se que as ocasiões disciplinares podem constituir ainda importante momento no esclarecimento sobre as expectativas parentais, proporcionando pistas afetivas e comportamentais dos pais em relação aos filhos (Baumrind, 1997; Silveira, Pacheco, Cruz, \& Schneider, 2005). Supomos que tais elementos afetivos, para a população brasileira, podem ter influenciado ou terem sido confundidas com o apoio emocional de conforto, proteção, afeto e o apoio no enfrentamento de situações estressantes, que caracteriza a dimensão de cuidado/apoio.

A fim de utilização desta escala padronizamos que itens com carga negativa dentro do fator devem ter seu valor invertido. Além disso, os itens dos fatores 1 e 3 trazem conteúdo de previsibilidade a partir de enunciados tais como "eu tinha certeza..." ou "eu sabia...", os valores destas dimensões precisam ser invertidos a fim de que reflitam imprevisibilidade, de acordo com as descrições das dimensões propostas por Ross e Hill $(2000,2002)$. Para fins de padronização de aplicação, a EIFI tem caráter autoaplicável ou de aplicação por meio de entrevista dependendo da escolaridade do participante.

Considerados os dados sociodemográficos dos participantes em relação às variáveis expectativa de vida e nível socioeconômico, apontadas na literatura como importantes na percepção de imprevisibilidade do ambiente (Hill et al., 2008; Hill et al., 1997), indicaram maior propensão de imprevisibilidade para grupo de adultos em conflito com a lei apresentando as menores expectativas de vida e renda. A EIFI seguiu a mesma tendência discriminativa identificando o grupo de adultos em conflito com a lei como o grupo com maiores médias de imprevisibilidade na infância (três das quatro dimensões possíveis) e diferenciando o mesmo nas dimensões de imprevisibilidade de recursos financeiros e imprevisibilidade de disciplina.

Concluímos que a EIFI foi validada para a população brasileira acrescentando-se no cenário nacional como um instrumento para medida de imprevisibilidade familiar na infância podendo ser autoaplicada ou aplicada sob a forma de entrevista, dependendo da escolaridade do participante. $\mathrm{O}$ estudo fornece um instrumento com parâmetros positivos de validade, tanto de construto, quanto de face, conteúdo e discriminante e elementos de confiabilidade para o emprego em pesquisas sobre imprevisibilidade familiar na infância no Brasil.

A validação possui a limitação de ter sido realizada no sudeste do Brasil podendo não contemplar diferentes formas de criação familiar e percepção desta criação presentes na diversidade da população brasileira. Sugere-se que estudos confirmatórios posteriores contemplem diver- 
sidades regionais de forma a discutir mais detalhadamente diferenças culturais referentes às dimensões disciplina $\mathrm{e}$ cuidado/apoio e confirmar a generalidade da escala para a população brasileira.

\section{Referências}

Alarcão, M., \& Gaspar, M. F. (2007). Imprevisibilidade familiar e suas implicações no desenvolvimento individual e familiar. Paidéia, 17(36), 89-102.

Baumrind, D. (1997). The discipline encounter: Contemporary issues. Agression and Violent Behavior, 2, 321-335.

Bernstein, D., \& Fink, L. (1998). Childhood trauma questionnaire: A retrospective self-report. San Antonio, TX: The Psychological Corporation.

Bernstein, D. P., Stein, J. A., Newcomb, M. D., Walker, E., Pogge, D., Ahluvalia, T., et al. (2003). Development and validation of a brief screening version of the childhood trauma questionnaire. Child Abuse \& Neglect, 27(2), 169-190.

Boeckel, M. G., \& Castellá Sarriera, J. (2005) Análise fatorial do Questionário de Estilos Parentais (PAQ) em uma amostra de adultos jovens universitários. Psico USF, 10(1), 1-9.

Buri, J. R. (1991). Parental Authority Questionnaire. Journal of Personality Assesment, 57(1), 110-119.

Daly, M., \& Wilson, M. (2001). Risk-taking, intrasexual competition, and homicide. Nebraska Symposium on Motivation, 47, 1-36.

Dancey, C. P., \& Reidy, J. (2006). Estatística sem matemática para psicologia usando SPSS para Windows. Porto Alegre, RS: Artes Médicas.

Erthal, T. C. (2001). Manual de Psicometria. Rio de Janeiro, RJ: Jorge Zahar.

Grassi-Oliveira, R., Stein, L. M., \& Pezzi, J. C. (2006). Tradução e validação de conteúdo da versão em português do Childhood Trauma Questionnaire. Revista de Saúde Pública, 40(2), 249-55.

Hill, E. M., Jenkins, J., \& Farmer, L. (2008). Family unpredictability, future discounting, and risk taking. The Journal of Socio-Economics, 37, 1381-1396.

Hill, E. M., Ross, L. T., \& Low, B. (1997). The role of future unpredictability in human risk-taking. Human Nature, 8 , 287-325.

Laros, J. A. (2005). O uso da análise fatorial: Algumas diretrizes para pesquisadores. In L. Pasquali (Ed.), Análise fatorial para pesquisadores (pp. 163-184). Brasília, DF: Editora da Universidade de Brasília.

Marturano, E. M. (2006). O inventário de recursos do ambiente familiar. Psicologia: Reflexão e Crítica, 19(3), 498-506.

Milfont, T. L., Gouveia, V. V., \& Costa, J. B. (2006). Determinantes psicológicos da intenção de constituir família. Psicologia: Reflexão e Crítica, 19(1), 25-33.

Miller, G. F. (1996). Protean primates: The evolution of adaptive unpredictability in competition and courtship. In A. Whiten \& R. Byrne (Eds.), Machiavellian intelligence II (pp. 312340). Cambridge, UK: Cambridge University Press.

Milliken, F. J. (1987). Three types of perceived uncertainty about the environment: State, effect, and response uncertainty. Academy of Management Review, 12, 133-143.

Pasquali, L. (1998). Princípios de elaboração de escalas psicológicas. Revista de Psiquiatria Clínica, 25(5), 206-213.

Pasquali, L. (1999). Instrumentos psicológicos: Manual prático de elaboração. Brasília, DF: Laboratório de Pesquisa em Avaliação e Medida.
Pasquali, L. (2001) Parâmetros psicométricos dos testes psicológicos. In L. Pasquali (Ed.), Técnicas de exame psicológico: Fundamentos das técnicas de exame psicológico (pp. 111-136). São Paulo, SP: Casa do Psicólogo.

Pasquali, L. (2005). Extração dos fatores. In L. Pasquali (Ed.), Análise fatorial para pesquisadores (pp. 55-86). Brasília, DF: Laboratório de Pesquisa em Avaliação e Medida.

Pasquali, L. (2007). Validade dos testes psicológicos: Será possível reencontrar o caminho? [Special issue]. Psicologia: Teoria e Pesquisa, 23, 99-107.

Pelzer, M. T., \& Fernandes, M. R. (1997). Apoiando a família que cuida de seu familiar idoso com demência. Texto Contexto Enfermagem, 6(3), 39-44.

Radimer, K. L., Olson, C. M., Greene, J. C., Campbell, C. C., \& Habicht, J. P. (1992). Understanding hunger and developing indicators to assess it in women and children. Journal of Nutrition Education, 24(1), 36-44.

Ross, L. T., \& Hill, E. M. (1995). Unpredictability schemas and problematic drinking. Paper presented at the meeting of Research Society on Alcoholism, Steamboat Springs, CO.

Ross, L. T., \& Hill, E. M. (2000). The Family Unpredictability Scale: Reliability and validity. Journal of Marriage and the Family, 62, 549-562.

Ross, L. T., \& Hill, E. M. (2002). Childhood unpredictability, schemas for future unpredictability, and risk taking. Social Behavior and Personality, 30, 453-474.

Ross, L. T., \& McDuff, J. A. (2008). The Retrospective Family Unpredictability Scale: Reliability and validity. Journal of Child Family Studies, 17, 13-27.

Segall-Corrêa, A. M., Pérez-Escamilla, R., Maranha, L. K., Sampaio, M. F. A., Yuyama, L., Alencar, F., et al. (2003). Projeto, acompanhamento e avaliação da segurança alimentar de famílias brasileiras: Validação de metodologia e de instrumento de coleta de informação (Relatório técnico). Campinas, SP: Universidade Estadual de Campinas, Departamento de Medicina Preventiva e Social.

Silveira, L. M. O. B., Pacheco, J., Cruz, T., \& Schneider, A. A. (2005). Estratégias educativas desejáveis e indesejáveis: Uma comparação entre a percepção de pais e mães de adolescentes. Aletheia, 21, 31-42.

Steinberg, L. (2004). Risk-taking in adolescence: What changes, and why? Annals of the New York Academy of Sciences, 1021, 51-58.

Urbina, S. (2007). Fundamentos da testagem psicológica. Porto Alegre, RS: Artmed. 